\section{HPV vaccine moves into late stage trials}

Until recently, the quest for a vaccine that protects against human papillomavirus (HPV) has been daunting. The virus has been difficult to grow in cultured cells; its link to cervical cancer took years to establish; new strains are continually being identified and now number more than 100; and clinical trials are difficult to conduct.

Fortunately, one single strain, HPV 16 , accounts for half of the cervical cancer that develops in the 400,000 or so women worldwide each year. By focusing on this single strain, researchers at the National Cancer Institute (NCI) and Johns Hopkins School of Public Health have used breakthrough technology to develop and test a virus-like particle (VLP) vaccine with promising results.

Although Merck and GlaxoSmithKline are also testing VLP vaccines for HPV, the NCI is the first to publish Phase I results on HPV 16.

"We are very encouraged," says John Schiller, senior investigator at the NCI. "So far the vaccine has been remarkably free of side effects." It also has proven to be highly immunogenic. The majority of 72 women receiving the vaccine achleved serum antibody titers nearly 40-fold higher than that found in natural infection. This level of antibody generation is impressive considering that HPV, unlike most viruses, results in no systemic infection. The virus evades immune survellance by shedding to external surfaces, where infection is manifest as lesions or warts.

As a proof-of-principal trial, the NCI study is testing whether this type of vaccine can protect against cancercausing HPV infection and cytological abnormalities. A single strain vaccine, for HPV 16 alone, is not commercially viable. However, including the other, less common strains numbered 18,31 and $\mathbf{4 5}$ that account for another quarter of cervical cancer-would make trials prohibitively large.

If the published results are confirmed in a recently completed Phase II study in the United States and Costa Rica, a full phase III trial in Costa Rica involving 10,000 women could begin this fall, Schiller says. So far, preliminary immunological analysis of the Phase II study looks as promising as the Phase I study, Schiller says. The five-year Phase III study will evaluate the protective effect of the single-strain vaccine through measuring persistent HPV DNA and low-grade squamous intraepithelial lesions (SIL), a precursor to cervical cancer. Using cervical cancer or even high-grade SIL would be unethical, Schiller notes.

The Merck vaccine, developed in collaboration with the Australian company, CSL, is in Phase II trials, according to spokesperson Carmen De Gourville. It is designed to cover four strains-16 and 18, the two most common cancer-causing strains, and 6 and 11 , which are not associated with cancer but account for most genital warts.

Adding types 6 and 11 to the vaccine will make trial time longer but this strategy is necessary to make a vaccine commercially viable, says Schiller. "Warts cause considerable discomfort and psycho-social trauma, so this makes the vaccine more attractive and also provides a reason other than altruism for men to be immunized," he explains.

Despite the progress, Schiller estimates that an approved vaccine is still five years away. Public health officials may need that much time to address the considerable social obstacles for what amounts to an "STD vaccine." Vaccination would begin in pre-adolescence, making it necessary for parents to discuss sex with their children. Even with the link between HPV and cervical cancer well established, many women with abnormal Pap tests aren't told they are likely infected with HPV.

"It can traumatize patients needlessly and yet it leaves a gap in the knowledge base for building demand for a vaccine," Schiller adds. For women already infected with HPV, the outlook also is promising. NCI is developing programs that will identify therapeutic vaccines effective against pre-malignant lesions. "You are going to see a lot of this in the future," he says. "It offers an opportunity to test ways of doing cancer immunotherapy because all these women are identified in Pap screening tests and you can monitor what is happening in a noninvasive fashion."

Harold Connett, Atlanta

\title{
Scientists march on parliament
}

Italian scientists are far from happy with their government's attitude toward genetic engineering research. As a demonstration of their discontent they have marched in front of the parliament building in protest. And in the run up to next month's general elections, they have sent a ten-point questionnaire to $\mathbf{4 4}$ political parties presenting them with precise questions on whether and how they would support such research and who should fund it. Responses will be published at http://guide.supereva.it

The ill-feelings were triggered by a February proposal from the agriculture minister, Alfonso Pecoraro Scanio, to restrict research on genetically modified organisms to a limited number of government-controlled laboratories. Scientists felt that this would block independent, commercial investment in biotechnology research.

The 1500-strong public protest-led by Nobel Laureate Rita Levi-Montalcini-is the first of its kind organized by Italy's scientific community, and has forced prime minister Giuliano Amato to intervene by commissioning a report on how the country plans to proceed with "safe" biotechnology research, which is due to be released this month. But many scientists dismiss this as method of stalling until votes have been cast in the election, which the centerright coalition is expected to win.

Scientist's hopes are pinned on Enrico Garaci, who is set to become head of the Higher Institute of Health this month. He told Nature Medicine that he opposes the restrictions on biotechnology research and plans to give acknowledged centers of excellence more funds.

Martina Ballmaier, Milan 